\title{
The magnetization profile induced by the double magnetic proximity effect in an Fe/Fe0.30V0.70 superlattice
}

H. Palonen, B. O. Mukhamedov, A. V. Ponomareva, G. K. Palsson, Igor Abrikosov and B. Hjorvarsson

The self-archived postprint version of this journal article is available at Linköping University Institutional Repository (DiVA):

http:// urn.kb.se/ resolve?urn=urn:nbn:se:liu:diva-159063

N.B.: When citing this work, cite the original publication.

Palonen, H., Mukhamedov, B. O., Ponomareva, A. V., Palsson, G. K., Abrikosov, I., Hjorvarsson, B., (2019), The magnetization profile induced by the double magnetic proximity effect in an Fe/ Fe0.30V0.70 superlattice, Applied Physics Letters, 115(1), 012406.

https:/ doi.org/ 10.1063/ 1.5102121

Original publication available at:

https:/ / doi.org/ 10.1063/ 1.5102121

Copyright: AIP Publishing

http:// www.aip.org/ 


\title{
The magnetization profile induced by the double magnetic proximity effect in an $\mathrm{Fe} / \mathrm{Fe}_{0.30} \mathrm{~V}_{0.70}$ superlattice
}

\author{
H. Palonen, ${ }^{1, \text { a) }}$ B. O. Mukhamedov, ${ }^{2}$ A. V. Ponomareva, ${ }^{2}$ G. K. Pálsson, ${ }^{1}$ I. A. Abrikosov, ${ }^{3}$ and B. Hjörvarsson ${ }^{1}$ \\ ${ }^{1)}$ Materials Physics, Department of Physics and Astronomy, Uppsala University, Box 530, SE-75121 Uppsala, \\ Sweden \\ ${ }^{2)}$ National University of Science \& Technology (MISIS), Materials Modeling and Development Laboratory, 119049 Moscow, \\ Russian Federation \\ 3) Department of Physics, Chemistry and Biology, Linköping University, SE-58183 Linköping, \\ Sweden
}

(Dated: 3 June 2019)

The double magnetic proximity effect in an $\mathrm{Fe} / \mathrm{Fe}_{0.30} \mathrm{~V}_{0.70}$ superlattice is studied by a direct measurement of the magnetization profile using polarized neutron reflectivity. The experimental magnetization profile is shown to qualitatively agree with a profile calculated with density functional theory. The profile is divided into a short range interfacial part and a long range tail. The interfacial part is explained by charge transfer and induced magnetization, while the tail is attributed to the inhomogeneous nature of the FeV alloy. The long range tail in the magnetization persists up to $170 \%$ above the intrinsic ordering temperature of the FeV alloy. The observed effects can be used to design systems with a direct exchange coupling between layers over long distances through a network of connected atoms. When combined with the recent advances in tuning and switching the MPE with electric fields and currents, the results can be applied in spintronic devices.

The magnetic equivalent of the superconducting proximity effect is the magnetic proximity effect (MPE) which manifests as a region of enhanced magnetization at an interface between two ferromagnets (FM) or between a FM and non-magnetic (NM) material. There are at least four different types of MPE that can occur: (i) In FM/NM systems the hybridization of electron orbitals leads to a charge transfer across the interface. If the charge transfer favours one spin state more than the other, the non-magnetic material will become locally spin polarized with an extension of few atomic layers. An example of charge transfer induced MPE is the Fe/V system where the first few $\mathrm{V}$ layers have a magnetic moment that is antiferromagnetically aligned with respect to the moment of the $\mathrm{Fe}$ layer ${ }^{1}$. (ii) There is an additional induced MPE at increased temperatures in a FM/FM system which can be rationalized by the high susceptibility of the weaker FM layer in the paramagnetic (PM) state above its ordering temperature ${ }^{2}$. (iii) If the NM layer of the FM/NM system is close to fulfilling the Stoner criterion, a strong MPE can be expected ${ }^{3-5}$. However, it is not always observed in such systems ${ }^{6,7}$. (iv) Magnetic heterostructures composed of alloys have been shown to exhibit large magnetic proximity effects, irrespectively of the crystalline ordering, for example, in amorphous CoAlZr alloys or in the crystalline $\mathrm{FeV}$ alloys of the present work ${ }^{8}$.

In this paper, the cases (i)-(ii) are referred to as the interfacial MPE and the case (iv) as chemical disorder MPE. A random alloy is not uniform in the atomic picture. Instead, there are regions with higher and lower chemical composition than the average. The regions make the alloy to be intrinsically inhomogeneous which is here referred to as chemical disorder.

An increased understanding of the origin of different types

\footnotetext{
a) Author to whom correspondence should be addressed: heikki.palonen@utu.fi
}

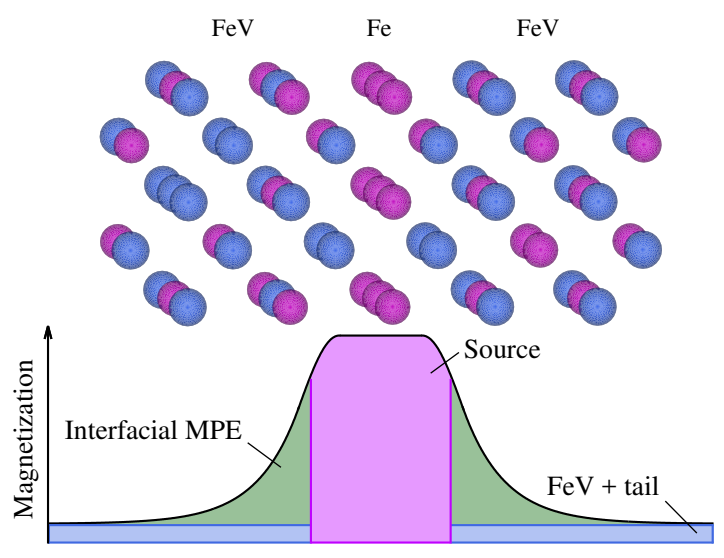

FIG. 1. A schematic of the sample structure (top) showing $1 \mathrm{ML}$ of $\mathrm{Fe}$ inserted inside an $\mathrm{FeV}$ alloy. A schematic of the magnetization profile (bottom) of the structure. The profile has been divided into regions (colours) that mark the different parts of the MPE.

of MPE is needed to have full control of the interface phenomena in magnetic heterostructures. For example, MPE is often accompanied by exchange bias, it is involved in the generation of spin currents in non-magnetic metals, and can be used to push up the ordering temperature of dilute magnetic semiconductors and topological insulators ${ }^{3,9-11}$. Furthermore, the MPE can be used to induce a moment above the Néel temperature in an antiferromagnetic system ${ }^{12}$. The relevance of MPE for applications is further emphasized by the recent findings of Yamada et al. and Koyama et al. who showed that the magnitude of the MPE can be tuned with electric field and that the magnetic moment in the MPE can be switched without switching the source ${ }^{13,14}$.

The double proximity effect is a combination of a finite size effect and an interfacial MPE resulting in a situation where a 

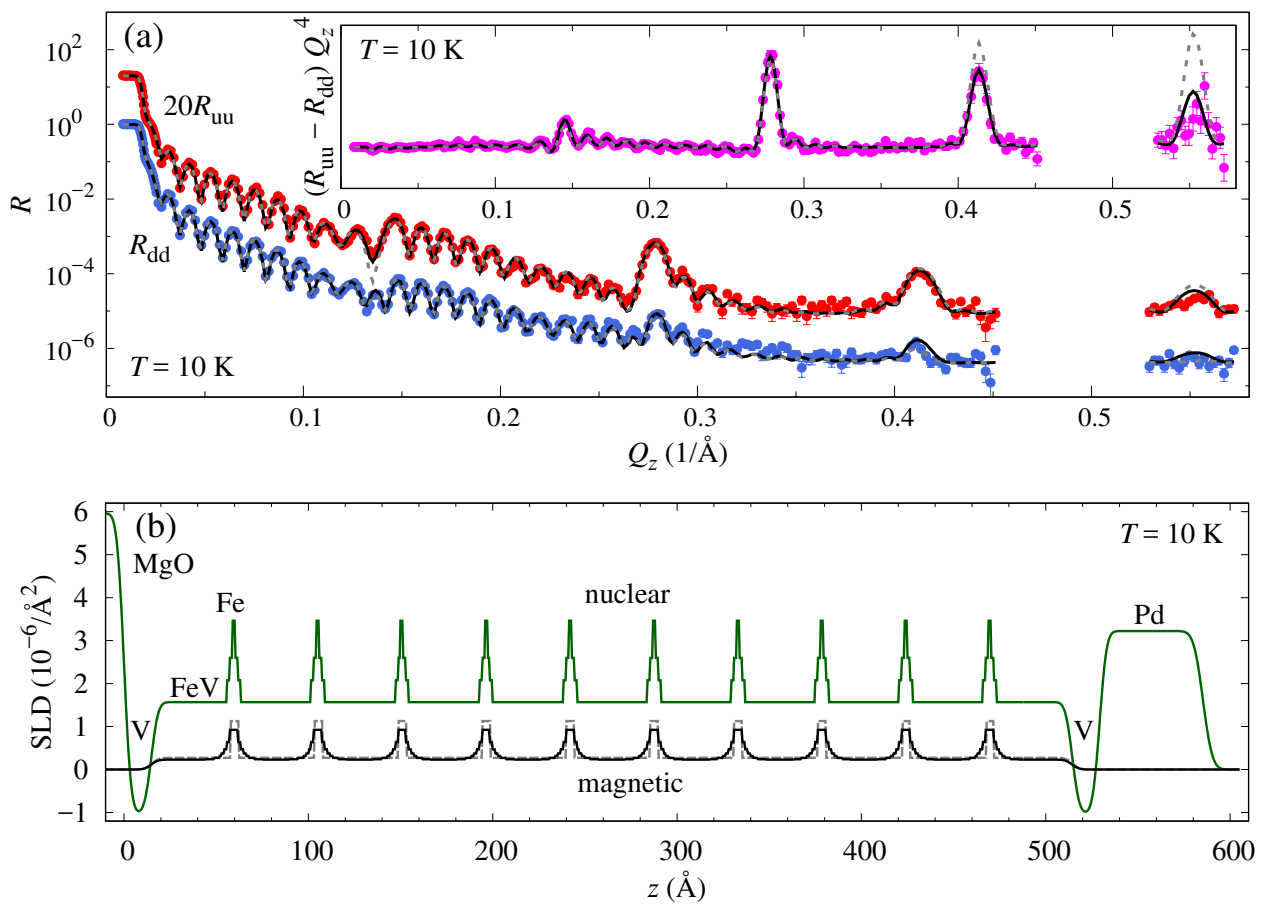

FIG. 2. (a) The PNR of the sample at $10 \mathrm{~K}$ shown on a logarithmic scale. The up-up reflectivity $\left(R_{\mathrm{uu}}\right)$ is shifted from the down-down reflectivity $\left(R_{\mathrm{dd}}\right)$ for clarity. The solid and dashed lines are the best fits with and without the interfacial MPE in the model, respectively. Inset: The difference of the up-up and down-down reflectivities scaled with $Q_{z}^{4}$ shown on linear scale. (b) The scattering length density (SLD) corresponding to the best fits with (solid) and without (dashed) the interfacial MPE. The nuclear SLD is determined by the elemental composition profile of the sample and the magnetic SLD by the magnetization profile. The up-up (down-down) reflectivity means that both the incident and reflected neutrons are spin up (down). The SLD corresponding to the up-up (down-down) reflectivity is the sum (difference) of the nuclear and magnetic SLD.

component is magnetic only because the other component is exerting MPE on it and vice versa. Previously, we have shown that there are strong double proximity effects in $\mathrm{Fe} / \mathrm{FeV}$ superlattices where the ordering temperature of the FeV alloy was doubled by the proximity of a single monolayer (ML) of Fe and the Fe layers were enhancing each other's ordering temperature across $30 \mathrm{ML}(4.5 \mathrm{~nm})$ of the $\mathrm{FeV}$ alloy ${ }^{2}$. While the long range of the exchange interaction between the Fe monolayers was demonstrated, not much could be concluded about the underlying mechanism using only average saturation magnetizations. Thus, direct measurements of the magnetization profile inside the structure are needed to gain more information on the mechanism.

In this paper, the focus is in the magnetization profile of a single $\mathrm{ML}$ of $\mathrm{Fe}$ in $\mathrm{Fe}_{0.30} \mathrm{~V}_{0.70}$ alloy which is schematically shown in Fig. 1. The profile is studied in a superlattice where the thickness of the alloy layer separating the Fe layers is $30 \mathrm{ML}$. The samples were grown by magnetron sputtering and the FeV alloy was done by co-sputtering. The FeV alloy composition ( 30 at. $\% \mathrm{Fe}$ ) was determined by measuring the ordering temperature $(60 \mathrm{~K})$ of the $\mathrm{FeV}$ reference alloy grown separately with the same growth parameters and comparing with the work of Mustaffa and Read ${ }^{15}$. More details of the sample preparation are available in the supplementary information (SI).
The magnetic moment of the bilayer can be divided into three parts: the magnetization of the source, a long tail of the magnetization extending far into the alloy together with the spontaneous magnetization of the alloy and the interfacial MPE region which decreases rapidly when moving away from the interface. The three regions are highlighted with different colours in Fig. 1. The interfacial MPE region arises from the charge transfer and high susceptibility of the alloy. If the tails of two nearest source layers overlap, the source layers will interact which leads to an increase of the ordering temperature.

The theoretical magnetization profile of the superlattice was calculated in the framework of the density functional theory (DFT) using the exact muffin-tin orbitals method (EMTO) combined with the coherent potential approximation ${ }^{16,17}$. The EMTO method uses the Green's function technique to solve the multiple scattering problem. The generalized gradient approximation was used to describe the exchange and correlation effects (see the SI for more details) ${ }^{18}$.

Experimentally, the magnetization profile in the $\mathrm{Fe} / \mathrm{FeV}$ superlattice can be measured by polarized neutron reflectivity (PNR) which gives a direct measurement of the spatial shape of the magnetic flux density in the sample. The PNR of the $\mathrm{Fe} / \mathrm{FeV}$ superlattice was measured at the SuperADAM reflectometer at the Institut Laue Langevin in Grenoble France ${ }^{19}$. The PNR data was fitted using GenX ${ }^{20}$. More details of the 
PNR experiment and the fitting procedure are available in the SI. Examples of the spin up-up, down-down reflectivities and their difference are shown in Fig. 2(a). The excellent quality of the measurement and the sample is emphasized by the fact that it was possible to measure very high in the $Q_{z}$, up to $0.55 \AA^{-1}$, where the fourth Bragg peak is still visible above the background.

A model that consists of the three parts shown as the coloured areas in Fig. 1 was used for fitting the measured PNR data. The magnetization of the source was approximated with a 3-ML-thick constant value. Considering the combination of the atomic steps smearing out the PNR results and the double peak structure near the Fe layer (to be discussed below), the approximation should be fairly good. In addition, the approach will be consistent with our previous work where the same approximation was used ${ }^{2}$. The interfacial MPE profile was modelled with an exponentially decreasing magnetization with increasing distance from the source. The exponential profile is to be expected if the interfacial MPE is considered to be induced by the magnetization of the neighbouring layer ${ }^{1,2}$. Only three free parameters were used in fitting the magnetization profile of the superlattice: the strength of the source, $M_{\delta}$, the spatially constant magnetization of the $\mathrm{FeV}$ layer, $M_{\mathrm{FeV}}$, and the characteristic length scale of the exponential function, $\xi$. The magnetization profile in the first half of the $\mathrm{FeV}$ layer is

$$
M(x)=\left(M_{\delta}-M_{\mathrm{FeV}}\right) \exp \left(-\frac{x}{\xi}\right)+M_{\mathrm{FeV}},
$$

where $x$ is the distance from the Fe layer interface. An example of the magnetization profile is shown in Fig. 2(b) together with the nuclear SLD of the superlattice. In Fig. 2 the solid (dashed) line shows the best fit and the corresponding SLD profile of the superlattice with (without) the interfacial MPE. Comparing the difference between the fits given by the models it can be seen that the model with the MPE included is more consistent with the data. Also, the first and the second order Bragg peaks are virtually unaffected by the shape of the magnetization profile near the Fe layer interface, which emphasizes the importance of collecting enough Fourier components to be able to measure interfacial MPE.

According to the DFT calculation, the average magnetic moment per atomic site in the superlattice has a maximum at the source, as can be seen in Fig. 3. A double peak structure can be seen in the source when looking at the moments per $\mathrm{Fe}$ atom which arises from the change of Fe coordination of the $\mathrm{Fe}$ atoms at the source layer: All the nearest neighbours of an atom inside a (100) body-centered cubic (bcc) monolayer are located in the neighbouring layers. As a result, the $\mathrm{Fe}$ atoms on the $\mathrm{FeV}$ side of the interface always have a higher Fe coordination than the $\mathrm{Fe}$ atoms in the single $\mathrm{ML}$ of $\mathrm{Fe}$, which leads to the double peak structure. The Fe moment reaches the bulk value of $2.2 \mu_{\mathrm{B}}$ on the alloy side of the interface while in the Fe ML it is only $1.2 \mu_{\mathrm{B}}$. The effects of the Fe coordination are also clearly visible in the charge transfer results that were calculated from the DFT (see the SI for the full charge transfer results). The $\mathrm{Fe}$ atoms on the $\mathrm{FeV}$ side of the interface have almost no charge transfer at all compared to the bulk Fe which

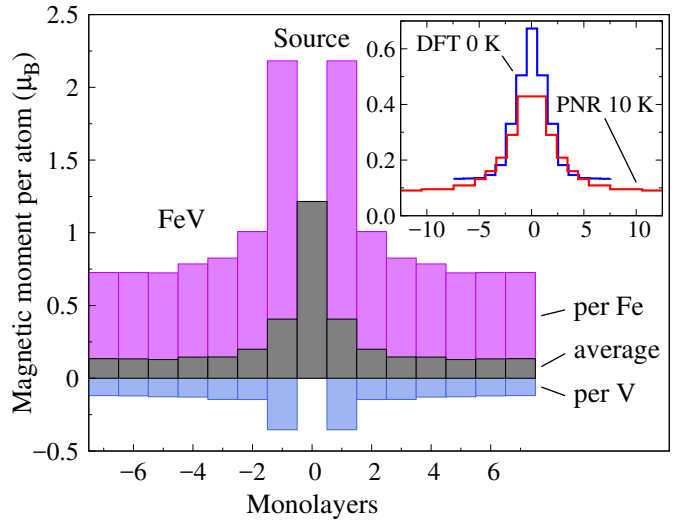

FIG. 3. The magnetic moment per atom and atomic site (average) given by the DFT calculation around the Fe ML that is the source. The negative values are used to indicate the antiferromagnetic alignment between the $\mathrm{Fe}$ and $\mathrm{V}$ moments. Inset: The magnetic moment per atomic site around one of the Fe layers according to the PNR fit at $10 \mathrm{~K}$ and according to the DFT calculation at $0 \mathrm{~K}$. The latter has been broadened with the nuclear SLD results to make the comparison more realistic. The units in the inset are the same as in the main figure.

leads to the high $\mathrm{Fe}$ moment. The $\mathrm{Fe}$ atoms in the Fe monolayer are strongly affected by charge transfer which is consistent with the weak (compared to bulk $\mathrm{Fe}$ ) moment of the $\mathrm{Fe}$ atoms in the monolayer. For the $\mathrm{V}$ at the interface, the theory predicts a moment of $0.35 \mu_{\mathrm{B}}$ which is antiferromagnetically aligned with respect to the $\mathrm{Fe}$ moments. The enhancement of the antiferromagnetic moment of the $\mathrm{V}$ atoms at the interface is also seen in the charge transfer results as a decrease of the $\mathrm{V}$ spin up electrons. The antiferromagnetic alignment and a moment of $0.7 \mu_{\mathrm{B}}$ for $\mathrm{V}$ at the interface has been previously reported in $\mathrm{Fe} / \mathrm{V}$ multilayers ${ }^{1}$. By comparison the DFT calculations underestimate the $\mathrm{V}$ moment. The range of the interfacial MPE around the source is about $3 \mathrm{ML}$ according to the calculation.

The PNR fit gives an average moment of $0.43 \mu_{\mathrm{B}}$ and $0.090 \mu_{\mathrm{B}}$ for the source and $\mathrm{FeV}$ layer, respectively, per atomic site at $10 \mathrm{~K}$. In our previous work based on vibrating sample magnetometry (VSM), a comparison between the samples resulted in $0.54 \mu_{\mathrm{B}}$ and $0.12 \mu_{\mathrm{B}}$ for the source and $\mathrm{FeV}$ layers ${ }^{2}$. The agreement is quite good considering that VSM gives only indirect information about the magnetization of individual layers, while PNR gives a direct measurement and that in this work the alloy has $30 \% \mathrm{Fe}$ instead of the $32 \%$ of the previous work.

The magnetization profiles given by the PNR fit and by DFT calculations around one of the source layers are shown in the inset of Fig. 3. To make the comparison between the theoretical and experimental curve more realistic, the DFT profile has been broadened by using the PNR fit result for the nuclear SLD. Since the total amount of $\mathrm{Fe}$ atoms in the grown Fe layer corresponds very accurately to what is needed to form a perfect monolayer, the nuclear SLD can be considered as a measure of how those atoms are distributed. This distribution 


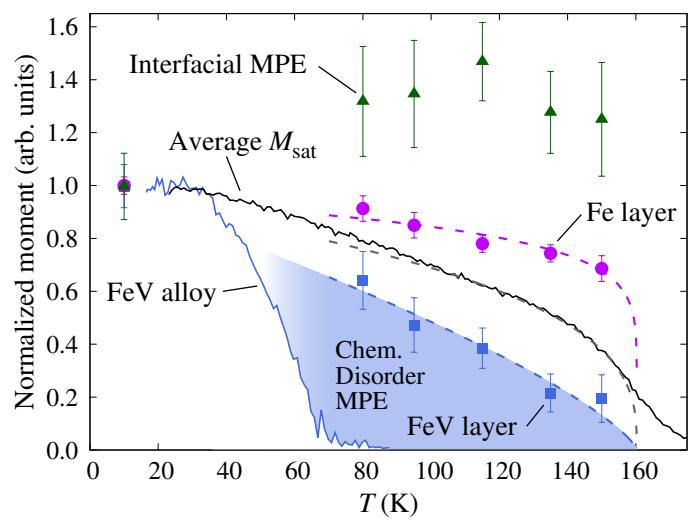

FIG. 4. The temperature dependence of the magnetic moments (points) in the different parts of the magnetization profile as illustrated in Fig. 1. The solid lines are the saturation magnetizations from the MOKE measurements of the superlattice sample and the reference $\mathrm{FeV}$ alloy with the same composition as was used in the $\mathrm{FeV}$ layer of the superlattice. The dashed lines are fits of eq. 2. The enhancement of the magnetic moment of the $\mathrm{FeV}$ alloy is marked with the coloured area.

is caused both by sample and instrumental effects; the measurement gives a one dimensional projection of the sample within the coherence volume. Atomic steps, for example, will smear out the resulting profile. The DFT result agrees qualitatively with the experimental profile but the absolute values are slightly overestimated which could be due to the underestimated V moments. The range of the interfacial MPE in the experimental result is not very long, only about $3 \mathrm{ML}$. Thus, the large MPE observed previously in the $\mathrm{Fe} / \mathrm{FeV}$ has to come from the enhancement of the spatially constant FeV layer moment instead of the interface ${ }^{2}$.

As mentioned above, the magnetization profile is divided into parts (see Fig. 1) which allows for further analysis by separately looking into the temperature dependencies of the regions. As is shown in Fig. 4, the Fe layer shows higher and the FeV layer smaller moments at all temperatures compared to the average moment. The moment of the FeV layer vanishes at the same temperature as the average moment showing that the system becomes fully ferromagnetic at a single ordering temperature. The moment in the interfacial MPE region does not decrease monotonically with increasing temperature because it has a large contribution coming from the magnetic susceptibility of the intrinsically paramagnetic alloy at the temperature region above the intrinsic ordering temperature of the FeV alloy.

The magnetization near the ordering temperature scales as

$$
M \propto(1-t)^{\beta}
$$

where $t$ is the reduced temperature and $\beta$ is an effective exponent. In this context, $\beta$ is not considered as a critical exponent since the system is not homogeneous. The moment of the source layer stays high even very close to the ordering temperature. The same behaviour was also observed in our previous work and is consistent with a two dimensional (2D) Ising system $(\beta=0.125)$ which is shown as the dashed line in Fig. 4.
The fact that the source layer is very thin and anisotropic is consistent with the $2 \mathrm{D}$ Ising behaviour ${ }^{2}$. The best fit gives an effective exponent of 0.74 and 0.34 for the $\mathrm{FeV}$ layer and the average magnetization measured by magneto-optical Kerr effect (MOKE), respectively. The latter two effective exponents should not be used to draw conclusions about the dimensionality because of the inhomogeneous nature of the system. The effective exponent of the FeV layer is high because of the temperature dependence of its magnetic susceptibility which is similar to what has been observed in $\mathrm{Fe} / \mathrm{Pd}^{4}$.

The nonvanishing and spatially constant value of the magnetization in the $\mathrm{FeV}$ between the source layers above the intrinsic ordering temperature of the alloy can be rationalized as a result of a slowly decreasing, almost linear, tail. Consider two tails that originate from the nearest Fe layers with slopes that are similar in magnitude but opposite in sign. When they are added up, the result is an approximately constant value. One possible origin for the long range tail is the chemical disorder of the FeV alloy: The randomness of the alloy is not homogeneous at the atomic scale which results in chains of connected Fe atoms where the other end of the chain is polarized and fixed by the continuous Fe layer. The chemical disorder is a universal property of random alloys and is likely to be the reason for the very long range MPE observed in the $\mathrm{Co}_{60} \mathrm{Al}_{28} \mathrm{Zr}_{12}$ as well ${ }^{8}$. Choi et al. have studied MPE in a $\mathrm{Gd} / \mathrm{Fe}$ system, which is free of chemical disorder ${ }^{21}$. The MPE that they observe is similar to the interfacial part of the MPE in our system but they observe no long range tail in the $\mathrm{Gd}$ layer which is consistent with our interpretation of the chemical disorder as the origin of the tail. The tail is not observed in the DFT calculations, because the local environment effects in a system with the chemical disorder would need to be included in the model of the alloy instead of working with the global average composition and because the DFT calculations are done at $0 \mathrm{~K}$, where the $\mathrm{FeV}$ alloy is ferromagnetic in any case.

A comparison of the $\mathrm{FeV}$ magnetization to the reference alloy shows that the MPE enhances strongly the ordering temperature of the $\mathrm{FeV}$, from $60 \mathrm{~K}$ to $160 \mathrm{~K}$ which is a $170 \%$ increase. The enhancement is marked with the coloured area in Fig. 4. The fact that the tail of the magnetization profile is constant across $30 \mathrm{ML}$ and that the constant part persists up to the ordering temperature, emphasizes the long range of the MPE at all temperatures.

In conclusion, the magnetization profile of the MPE in a FM/FM and FM/PM interface, where the PM system is above its intrinsic ordering temperature and has chemical disorder, can be rationalized to consist of an interfacial part and a slowly decreasing tail. The tail extends far from the interface while the interfacial part that originates from induced magnetization and charge transfer effects has a short range. Changes in the $\mathrm{Fe}$ coordination are the origin of the charge transfer effects. The strong induction is explained by the high magnetic susceptibility of the FeV alloy above the intrinsic ordering temperature of the alloy. The tail of the MPE is caused by the inhomogeneous nature of the $\mathrm{FeV}$ alloy resulting in an extended network of connected magnetic atoms. The tail has a long range and persists high, up to $170 \%$, above the in- 
trinsic ordering temperature of the alloy. The observed MPE enables additional tunability in the design of magnetic materials allowing for a direct exchange coupling over long distances through a network of connected atoms. Furthermore, the results are applicable in spintronics when they are combined with the recent advances in tuning and switching the MPE with electric fields and currents.

See the supplementary information for the complete description of the structure calculations, experimental methods, PNR fits and charge transfer results.

Financial support from the Swedish Research Council is gratefully acknowledged. The theoretical calculations were supported by the Ministry of Science and High Education of the Russian Federation in the framework of Increase Competitiveness Program of NUST (MISIS) (no. K2-2019-001) implemented by a governmental decree (16th March 2013, no. 211). Financial support from the Swedish Government Strategic Research Areas in Materials Science on Functional Materials at Linköping University (Faculty Grant SFO-MatLiU No. 2009-00971) and the Swedish e-Science Centre is gratefully acknowledged.

${ }^{1}$ M. A. Tomaz, W. J. Antel Jr, W. L. O'Brien, and G. R. Harp, "Induced $\mathrm{V}$ moments in Fe/V(100), (211), and (110) superlattices studied using Xray magnetic circular dichroism," J. Phys.: Condens. Matter 9, L179-L184 (1997).

${ }^{2}$ H. Palonen, F. Magnus, and B. Hjörvarsson, "Double magnetic proximity in $\mathrm{Fe} / \mathrm{Fe}_{0.32} \mathrm{~V}_{0.68}$ superlattices,” Phys. Rev. B 98, 144419 (2018).

${ }^{3}$ S. Y. Huang, X. Fan, D. Qu, Y. P. Chen, W. G. Wang, J. Wu, T. Y. Chen, J. Q. Xiao, and C. L. Chien, "Transport magnetic proximity effects in platinum," Phys. Rev. Lett. 109, 107204 (2012).

${ }^{4}$ T. P. A. Hase, M. S. Brewer, U. B. Arnalds, M. Ahlberg, V. Kapaklis, M. Björck, L. Bouchenoire, P. Thompson, D. Haskel, Y. Choi, J. Lang, C. Sánchez-Hanke, and B. Hjörvarsson, "Proximity effects on dimensionality and magnetic ordering in Pd/Fe/Pd trialyers," Phys. Rev. B 90, 104403 (2014).

${ }^{5}$ S. Cao, M. Street, J. Wang, J. Wang, X. Zhang, C. Binek, and P. A. Dowben, "Magnetization at the interface of Cr2O3and paramagnets with large stoner susceptibility,” J. Phys.: Condens. Matter 29, 10LT01 (2017).

${ }^{6}$ S. Geprägs, S. Meyer, S. Altmannshofer, M. Opel, F. Wilhelm, A. Rogalev, R. Gross, and S. T. B. Goennenwein, "Investigation of induced Pt magnetic polarization in $\mathrm{Pt} / \mathrm{Y}_{3} \mathrm{Fe}_{5} \mathrm{O}_{12}$ bilayers," Appl. Phys. Lett. 101, 262407 (2012).

${ }^{7}$ M. Collet, R. Mattana, J.-B. Moussy, K. Ollefs, S. Collin, C. Deranlot, A. Anane, V. Cros, F. Petroff, F. Wilhelm, and A. Rogalev, "Investigating magnetic proximity effects at ferrite/Pt interfaces," Appl. Phys. Lett. 111, 202401 (2017).

${ }^{8}$ F. Magnus, M. E. Brooks-Bartlett, R. Moubah, R. A. Procter, G. Andersson, T. P. A. Hase, S. T. Banks, and B. Hjörvarsson, "Long-range magnetic interactions and proximity effects in an amorphous exchange-spring magnet," Nat. Commun. 7, 11931 (2016).

${ }^{9}$ P. K. Manna and S. M. Yusuf, "Two interface effects: Exchange bias and magnetic proximity," Phys. Rep. 535, 61-99 (2014).

${ }^{10}$ C. Song, M. Sperl, M. Utz, M. Ciorga, G. Woltersdorf, D. Schuh, D. Bougeard, C. H. Back, and D. Weiss, "Proximity Induced Enhancement of the Curie Temperature in Hybrid Spin Injection Devices," Phys. Rev. Lett. 107, 056601 (2011).

${ }^{11}$ W. Liu, L. He, Y. Xu, K. Murata, M. C. Onbasli, M. Lang, N. J. Maltby, S. Li, X. Wang, C. A. Ross, P. Bencok, G. van der Laan, R. Zhang, and K. L. Wang, "Enhancing Magnetic Ordering in Cr-Doped $\mathrm{Bi}_{2} \mathrm{Se}_{3}$ Using High- $T_{\mathrm{C}}$ Ferrimagnetic Insulator," Nano Lett. 15, 764 (2015).

${ }^{12}$ S. M. Suturin, V. V. Fedorov, A. G. Banshchikov, D. A. Baranov, K. V. Koshmak, P. Torelli, J. Fujii, G. Panaccione, K. Amemiya, M. Sakamaki, T. Nakamura, M. Tabuchi, L. Pasquali, and N. S. Sokolov, "Proximity effects and exchange bias in $\mathrm{Co} / \mathrm{MnF} 2(111)$ heterostructures studied by $\mathrm{x}$-ray magnetic circular dichroism," J. Phys.: Condens. Matter 25, 046002 (2013).

${ }^{13}$ K. T. Yamada, M. Suzuki, A.-M. Pradipto, T. Koyama, S. Kim, K.-J. Kim, S. Ono, T. Taniguchi, H. Mizuno, F. Ando, K. Oda, H. Kakizakai, T. Moriyama, K. Nakamura, D. Chiba, and T. Ono, "Microscopic Investigation into the Electric Field Effect on Proximity-Induced Magnetism in Pt,” Phys. Rev. Lett. 120, 157203 (2018).

${ }^{14}$ T. Koyama, Y. Guan, Y. Hibino, M. Suzuki, and D. Chiba, "Magnetization switching by spin-orbit torque in Pt with proximity-induced magnetic moment," J. Appl. Phys. 121, 123903 (2017).

${ }^{15}$ A. Mustaffa and D. A. Read, "Magnetic properties of ferromagnetic VFe alloys near the critical concentration for ferromagnetism," J. Magn. Magn. Mater. 5, 349-352 (1977).

${ }^{16}$ L. Vitos, I. A. Abrikosov, and B. Johansson, "Anisotropic Lattice Distortions in Random Alloys from First-Principles Theory,” Phys. Rev. Lett. 87, 156401 (2001).

${ }^{17}$ L. Vitos, Computational Quantum Mechanics for Materials Engineers: the EMTO Method and Applications (Springer-Verlag, London, 2007).

${ }^{18}$ J. P. Perdew, K. Burke, and M. Ernzerhof, "Generalized Gradient Approximation Made Simple," Phys. Rev. Lett. 77, 3865 (1996).

${ }^{19}$ A. Vorobiev, A. Devishvilli, G. Palsson, H. Rundlöf, N. Johansson, A. Olsson, A. Dennison, M. Wollf, B. Giroud, O. Aguettaz, and B. Hjörvarsson, "Recent upgrade of the polarized neutron reflectometer Super ADAM," Neutron News 26, 25 (2015).

${ }^{20} \mathrm{M}$. Björck and G. Andersson, "GenX: an extensible X-ray reflectivity refinement program utilizing differential evolution,” J. Appl. Crystallogr. 40, 1174 (2007).

${ }^{21}$ Y. Choi, D. Haskel, R. E. Camley, D. R. Lee, J. C. Lang, G. Srajer, J. S. Jiang, and S. D. Bader, "Temperature evolution of the Gd magnetization profile in strongly coupled Gd/Fe multilayers," Phys. Rev. B 70, 134420 (2004). 